\title{
Respiratory Review of 2014: Asthma
}

\author{
Ho Il Yoon, M.D., Ph.D.
}

Department of Internal Medicine, Seoul National University College of Medicine, Seoul, Korea

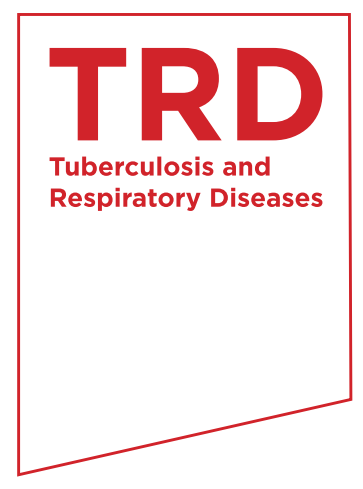

Asthma is a chronic inflammatory disease of the airway that comprises a variety of etiologies and inflammatory phenotypes. Clinically, there is a wide range of patients with varying severities and responses to individual drugs. The introduction of inhaled corticosteroid therapy has dramatically changed the treatment of asthma. Recent development of new therapies suggests the possibility of another breakthrough. These can be categorized as follows: anti-cytokine therapies that usually target eosinophilic inflammation, sublingual immunotherapy, and bronchial thermoplasty. In this paper, we will review the major articles related to asthma treatment that were published in 2013.

Keywords: Asthma; Eosinophilia; Cytokines; Sublingual Immunotherapy

\section{Dupilumab in persistent asthma with elevated eosino- phil levels. Wenzel et al. ${ }^{1}$ N Engl J Med 2013;368:2455- 66}

Background Moderate-to-severe asthma remains poorly treated. We evaluated the efficacy and safety of dupilumab (SAR231893/REGN668), a fully human monoclonal antibody to the alpha subunit of the interleukin-4 receptor, in patients with persistent, moderate-to-severe asthma and elevated eosinophil levels.

Methods We enrolled patients with persistent, moderate-tosevere asthma and a blood eosinophil count of at least 300 cells per microliter or a sputum eosinophil level of at least $3 \%$ who used medium-dose to high-dose inhaled glucocorticoids plus long-acting beta-agonists (LABAs). We administered dupilumab (300 mg) or placebo subcutaneously once weekly.

Address for correspondence: Ho II Yoon, M.D., Ph.D.

Department of Internal Medicine, Seoul National University College of Medicine, 103 Daehak-ro, Jongno-gu, Seoul 110-799, Korea

Phone: 82-31-787-7036, Fax: 82-31-787-7041

E-mail: dextro70@gmail.com

Received: Nov. 17, 2014

Revised: Nov. 25, 2014

Accepted: Dec. 1, 2014

(c) It is identical to the Creative Commons Attribution Non-Commercial License (http://creativecommons.org/licenses/by-nc/3.0/).

Copyright (c) 2014

The Korean Academy of Tuberculosis and Respiratory Diseases.

All rights reserved.
Patients were instructed to discontinue LABAs at week 4 and to taper and discontinue inhaled glucocorticoids during weeks 6 through 9. Patients received the study drug for 12 weeks or until a protocol-defined asthma exacerbation occurred. The primary end point was the occurrence of an asthma exacerbation; secondary end points included a range of measures of asthma control. Effects on various type 2 helper T-cell (Th2)-associated biomarkers and safety and tolerability were also evaluated.

Results A total of 52 patients were assigned to the dupilumab group, and 52 patients were assigned to the placebo group. Baseline characteristics were similar in the two groups. Three patients had an asthma exacerbation with dupilumab (6\%) versus 23 with placebo (44\%), corresponding to an $87 \%$ reduction with dupilumab (odds ratio, 0.08 ; $95 \%$ confidence interval, 0.02 to 0.28 ; $\mathrm{P}<0.001$ ). Significant improvements were observed for most measures of lung function and asthma control. Dupilumab reduced biomarkers associated with Th2-driven inflammation. Injection-site reactions, nasopharyngitis, nausea, and headache occurred more frequently with dupilumab than with placebo.

Conclusions In patients with persistent, moderate-to-severe asthma and elevated eosinophil levels who used inhaled glucocorticoids and LABAs, dupilumab therapy, as compared with placebo, was associated with fewer asthma exacerbations when LABAs and inhaled glucocorticoids were withdrawn, with improved lung function and reduced levels of Th2-associated inflammatory markers. (Wenzel et al. ${ }^{1}$, 2013, p. 2455; Reprinted with permission of Massachusetts Medical Society)

The effect of administration of dupilumab (SAR31893/ REGN668), a human monoclonal antibody of alpha subunit 
of interleukin (IL)-4 receptor, on the treatment of moderate to severe asthma with increased eosinophils was analyzed. Patients with moderate to severe persistent asthma despite treatments with high doses of inhaled steroids (ICSs) and long acting beta agonists (LABA) and those who showed peripheral blood eosinophil count more than $300 / \mathrm{mcl}$ or sputum eosinophils is more than $3 \%$ were randomized to 12 weeks of therapy with dupilumab or placebo. Fifty-two patients were assigned in each group and there were statistically significant difference (6\% in dupilumab and $44 \%$ in the placebo group) in exacerbation rates, which was the primary outcome. In addition, the effect has been demonstrated also in terms of lung function and symptoms improvement. Biomarkers such as fractional exhaled nitric oxide, thymus and activation regulated chemokine, eotaxin-3, IgE levels have proved to be significantly reduced. Various side effects such as injection site pain, nasopharyngitis, nausea, and headache have been reported.

Potentially useful drug in the treatment of severe asthma that is not well controlled with current drugs. However, the external validity is limited because we cannot know the effect of this drug on patients without eosinophilia.

Randomized, double-blind, placebo-controlled study of brodalumab, a human anti-IL-17 receptor monoclonal antibody, in moderate to severe asthma. Busse et al. ${ }^{2}$ Am J Respir Crit Care Med 2013;188:1294-302

Rationale: IL-17 signaling has been implicated in development and persistence of asthma. Cytokine-targeted strategies blocking IL-17 receptor signaling may be beneficial in asthma treatment.

Objectives: To determine efficacy and safety of brodalumab, a human anti-IL-17 receptor A monoclonal antibody, in subjects with inadequately controlled moderate to severe asthma taking regular inhaled corticosteroids.

Methods: Three hundred two subjects were randomized to brodalumab $(140,210$, or $280 \mathrm{mg}$ ) or placebo. Primary endpoint was change in Asthma Control Questionnaire (ACQ) score from baseline to Week 12. Secondary endpoints included FEV1, symptom scores, and symptom-free days. Prespecified subgroup analyses were conducted to identify potential responsive subpopulations. Analyses included randomized subjects receiving one or more doses of investigational product using last-observation-carried-forward imputation.

Measurements and Main Results: Demographics and baseline characteristics were generally balanced among groups ( $n=302$; $\mathrm{n}=226$ brodalumab). For the overall study population, no treatment differences were observed. Nine prespecified subgroups were examined without corrections for multiple testing. In only the high-reversibility subgroup (post-bronchodilator FEV1 improvement $\geq 20 \% ; n=112$ ) was an ACQ change with nominal significance noted; ACQ responses were nominally significant in the 210-mg group (estimated treatment difference, 0.53 ) but not significant in the higher 280-mg group (estimated treatment difference, 0.38). Adverse events, generally balanced among groups, were most commonly asthma, upper respiratory tract infection, and injection site reaction.

Conclusions: Inhibition of IL-17 receptor A did not produce a treatment effect in subjects with asthma. The results of the highreversibility subgroup analysis are of uncertain significance, requiring further study of brodalumab in this asthma subpopulation. (Busse et al. ${ }^{2}, 2013$, p. 1294, Reprinted with permission of the American Thoracic Society. Copyright (C2014 American Thoracic Society. Official Journal of the American Thoracic Society)

IL-17 has also been known to contribute to the generation and maintenance of asthma. This study is to examine the effects of brodalumab (human monoclonal antibody against IL17 receptor A) on the treatment of severe asthma that is not well controlled with current mediation. Three hundred and two patients were divided into four groups (140 mg, $210 \mathrm{mg}$, $280 \mathrm{mg}$, and placebo for 12 weeks), and the primary outcome was asthma control questionnaire (Asthma Control Questionnaire $[A C Q])$. There were no statistically significant differences in ACQ, forced expiratory volume in one second $\left(\mathrm{FEV}_{1}\right)$, and symptom free days.

Effects of benralizumab on airway eosinophils in asthmatic patients with sputum eosinophilia. Laviolette et al. ${ }^{3}$ J Allergy Clin Immunol 2013;132:1086-96

BACKGROUND: Many asthmatic patients exhibit sputum eosinophilia associated with exacerbations. Benralizumab targets eosinophils by binding IL-5 receptor $\alpha$, inducing apoptosis through antibody-dependent cell-mediated cytotoxicity.

OBJECTIVES: We sought to evaluate the safety of benralizumab in adults with eosinophilic asthma and its effects on eosinophil counts in airway mucosal/submucosal biopsy specimens, sputum, bone marrow, and peripheral blood.

METHODS: In this multicenter, double-blind, placebo-controlled phase I study, 13 subjects were randomized to singledose intravenous placebo or $1 \mathrm{mg} / \mathrm{kg}$ benralizumab (day 0; cohort 1), and 14 subjects were randomized to 3 monthly subcutaneous doses of placebo or 100 or $200 \mathrm{mg}$ of benralizumab (days 0, 28, and 56; cohort 2). Cohorts 1 and 2 were consecutive.

RESULTS: The incidence of adverse events was similar between groups. No serious adverse events related to benralizumab occurred. In cohort 1 intravenous benralizumab produced a median decrease from baseline of $61.9 \%$ in airway mucosal eosinophil counts (day 28; placebo: $+19.6 \%$; $\mathrm{P}=.28$ ), as well as an $18.7 \%$ decrease (day 21) in sputum and a $100 \%$ decrease (day 28) in blood counts. Eosinophils were not detectable in bone marrow of benralizumab-treated subjects (day 28, n = 4). In cohort 2 subcutaneous benralizumab demonstrated a combined 
$(100+200 \mathrm{mg})$ median reduction of $95.8 \%$ in airway eosinophil counts (day 84; placebo, $46.7 \%$; $\mathrm{P}=.06$ ), as well as an $89.9 \%$ decrease (day 28) in sputum and a $100 \%$ decrease (day 84 ) in blood counts.

CONCLUSION: Single-dose intravenous and multiple-dose subcutaneous benralizumab reduced eosinophil counts in airway mucosa/submucosa and sputum and suppressed eosinophil counts in bone marrow and peripheral blood. The safety profile supports further development. Additional studies are needed to assess the clinical benefit in asthmatic patients. (Laviolette et al. ${ }^{3}$, 2013, p. 1086; Reprinted with permission of Elsevier)

Benralizumab is a monoclonal antibody against IL-5 receptor alpha. The present study was conducted to evaluate the efficacy and safety of benralizumab in treating asthma patients with eosinophilic inflammation. Although it was a phase I clinical trial, but even though, by proving effectively suppressing eosinophilic inflammation with only a single infusion followed by subcutaneous injections, these results increase the prospect of practical use of anti-cytokine therapies.

Sublingual immunotherapy for the treatment of allergic rhinoconjunctivitis and asthma: a systematic review. Lin et al. ${ }^{4}$ JAMA 2013;309:1278-88

Importance Allergic rhinitis affects up to $40 \%$ of the US population. To desensitize allergic individuals, subcutaneous injection immunotherapy or sublingual immunotherapy may be administered. In the United States, sublingual immunotherapy is not approved by the Food and Drug Administration. However, some US physicians use aqueous allergens, off-label, for sublingual desensitization.

Objective To systematically review the effectiveness and safety of aqueous sublingual immunotherapy for allergic rhinoconjunctivitis and asthma.

Evidence Acquisition The databases of MEDLINE, EMBASE, LILACS, and the Cochrane Central Register of Controlled Trials were searched through December 22, 2012. English-language randomized controlled trials were included if they compared sublingual immunotherapy with placebo, pharmacotherapy, or other sublingual immunotherapy regimens and reported clinical outcomes. Studies of sublingual immunotherapy that are unavailable in the United States and for which a related immunotherapy is unavailable in the United States were excluded. Paired reviewers selected articles and extracted the data. The strength of the evidence for each comparison and outcome was graded based on the risk of bias (scored on allocation, concealment of intervention, incomplete data, sponsor company involvement, and other bias), consistency, magnitude of effect, and the directness of the evidence.

Results Sixty-three studies with 5131 participants met the inclusion criteria. Participants' ages ranged from 4 to 74 years.
Twenty studies ( $\mathrm{n}=1814$ patients) enrolled only children. The risk of bias was medium in 43 studies (68\%). Strong evidence supports that sublingual immunotherapy improves asthma symptoms, with 8 of 13 studies reporting greater than $40 \%$ improvement vs the comparator. Moderate evidence supports that sublingual immunotherapy use decreases rhinitis or rhinoconjunctivitis symptoms, with 9 of 36 studies demonstrating greater than $40 \%$ improvement vs the comparator. Medication use for asthma and allergies decreased by more than $40 \%$ in 16 of 41 studies of sublingual immunotherapy with moderate grade evidence. Moderate evidence supports that sublingual immunotherapy improves conjunctivitis symptoms (13 studies), combined symptom and medication scores (20 studies), and disease-specific quality of life (8 studies). Local reactions were frequent, but anaphylaxis was not reported.

Conclusions and Relevance The overall evidence provides a moderate grade level of evidence to support the effectiveness of sublingual immunotherapy for the treatment of allergic rhinitis and asthma, but high-quality studies are still needed to answer questions regarding optimal dosing strategies. There were limitations in the standardization of adverse events reporting, but no life-threatening adverse events were noted in this review. (Lin et al. ${ }^{4}, 2013$, p. 1278; Reprinted with permission of American Medical Association)

A systematic review on the effect of sublingual immunotherapy on the treatment of asthma was done. Included 5,131 patients from 63 studies were analyzed that sublingual immunotherapy improves symptoms of asthma (more than $40 \%$ in 8 out of 13 studies), reduces the use of asthma medications (decreased more than $40 \%$ in 16 out of 41 studies), and improves the quality of life. Based these results the authors claim that sublingual immunotherapy is supported by evidence to be effective in treatment of asthma.

Comparative outcomes of leukotriene receptor antagonists and long-acting $\beta$-agonists as add-on therapy in asthmatic patients: a population-based study. Sadatsafavi et al. ${ }^{5}$ J Allergy Clin Immunol 2013;132:63-9

BACKGROUND: Recent evidence suggests that the use of leukotriene receptor antagonists (LTRAs) in addition to inhaled corticosteroids (ICSs) in asthmatic patients provides comparable benefits to the addition of long-acting $\beta$-agonists (LABAs) to ICSs.

OBJECTIVE: We sought to compare, in a unified framework, adherence, outcomes, and costs associated with ICS+LTRA versus ICS+LABA as step-up therapies for asthma.

METHODS: Using the administrative databases of British Columbia, Canada (years 1997-2007), we created a propensity score-matched sample of asthmatic patients (12-45 years old) receiving ICS+LTRA therapy versus ICS+LABA therapy after a period of monotherapy with an ICS. We compared the out- 
comes using 2 analyses: an intention-to-treat (ITT) analysis that followed subjects for a fixed period of 2 years and an uninterrupted treatment analysis that followed subjects for as long as they continuously dispensed their index medications.

RESULTS: The matched cohort consisted of 1032 subjects in each group (mean age at entry, 27.4 years; $52.5 \%$ female). Adherence, which was defined as the proportion of days covered, was higher in the ICS+LABA group compared with the ICS+LTRA group. In both the ITT and uninterrupted treatment analyses, use of ICS+LTRA therapy was associated with more asthma-related outpatient visits, asthma-related medication dispensations, and dispensation of reliever medications. Dispensation of oral corticosteroids and rate of asthma exacerbations were higher in the ICS+LTRA group in the uninterrupted treatment analysis but not in the ITT analysis.

CONCLUSIONS: In a real-world clinical setting subjects were more adherent to ICS+LABA therapy than ICS+LTRA therapy. ICS+LABA therapy seems to be more effective than ICS+LTRA therapy in the management of asthma, regardless of adherence. (Sadatsafavi et al. ${ }^{5}$ 2013, p. 63; Reprinted with permission of Elsevier)

A retrospective cohort study to compare adding leukotriene antagonists (LTRA) to ICSs and adding LABA to ICS in terms of patient compliance, treatment outcome, and cost. The authors conclude that the ICS+LABA group was superior to the ICS+LTRA group after analyzing 2,000 patients during 11 years since 1997 in Canada. This study has all the limitations that retrospective studies might have, but also reflects some realities of real clinical world.

Bronchial thermoplasty: long-term safety and effectiveness in patients with severe persistent asthma. Wechsler et al. ${ }^{6}$ J Allergy Clin Immunol 2013;132:1295302

BACKGROUND: Bronchial thermoplasty (BT) has previously been shown to improve asthma control out to 2 years in patients with severe persistent asthma.

OBJECTIVE: We sought to assess the effectiveness and safety of BT in asthmatic patients 5 years after therapy.

METHODS: BT-treated subjects from the Asthma Intervention Research 2 trial (ClinicalTrials.govNCT01350414) were evaluated annually for 5 years to assess the long-term safety of BT and the durability of its treatment effect. Outcomes assessed after BT included severe exacerbations, adverse events, health care use, spirometric data, and high-resolution computed tomographic scans.

RESULTS: One hundred sixty-two (85.3\%) of 190 BT-treated subjects from the Asthma Intervention Research 2 trial completed 5 years of follow-up. The proportion of subjects experiencing severe exacerbations and emergency department (ED) visits and the rates of events in each of years 1 to 5 remained low and were less than those observed in the 12 months before BT treatment (average 5-year reduction in proportions: $44 \%$ for exacerbations and $78 \%$ for ED visits). Respiratory adverse events and respiratory-related hospitalizations remained unchanged in years 2 through 5 compared with the first year after BT. Prebronchodilator $\mathrm{FEV}_{1}$ values remained stable between years 1 and 5 after BT, despite a 18\% reduction in average daily inhaled corticosteroid dose. High-resolution computed tomographic scans from baseline to 5 years after BT showed no structural abnormalities that could be attributed to BT.

CONCLUSIONS: These data demonstrate the 5-year durability of the benefits of BT with regard to both asthma control (based on maintained reduction in severe exacerbations and ED visits for respiratory symptoms) and safety. BT has become an important addition to our treatment armamentarium and should be considered for patients with severe persistent asthma who remain symptomatic despite taking inhaled corticosteroids and long-acting $\beta 2$-agonists. (Wechsler et al. ${ }^{6}, 2013$, p. 1295; Reprinted with permission of Elsevier)

After bronchial thermoplasty (BT) has announced the result that BT is helpful in asthma control there has been concerns about the duration of the effect and long-term sequelae. This research is a study evaluating the efficacy and safety after 5 years in patients with asthma who underwent BT. They have analysed the 162 participants in the Asthma Intervention Research 2 study. Severe deterioration or emergency room visits were maintained low for up to 5 years. $\mathrm{FEV}_{1}$ has not changed up to 5 years and high-resolution computer computerized tomography scan results showed no structural abnormalities. The authors argue that the sustainability and long-term safety of BT has been demonstrated in the treatment of severe persistent asthma.

Inhaled corticosteroids and the risk of pneumonia in people with asthma: a case-control study. McKeever et al. ${ }^{7}$ Chest 2013;144:1788-94

BACKGROUND: In clinical trials, the use of inhaled corticosteroids is associated with an increased risk of pneumonia in people with COPD, but whether the same is true for people with asthma is not known.

METHODS: With the use of primary care data from The Health Improvement Network, we identified people with asthma, and from this cohort, we identified patients with pneumonia or lower respiratory tract infection and age- and sex-matched control subjects. Conditional logistic regression was used to determine the association between the dose and type of inhaled corticosteroid and the risk of pneumonia or lower respiratory tract infection.

RESULTS: A dose-response relationship was found between the strength of inhaled corticosteroid dose and risk of pneumonia or lower respiratory tract infection (P \&lt; .001 for trend) 
such that after adjusting for confounders, people receiving the highest strength of inhaled corticosteroid $(\geq 1,000 \mu \mathrm{g})$ had a 2.04 (95\% CI, 1.59-2.64) increased risk of pneumonia or lower respiratory tract infection compared with those with asthma who did not have a prescription for inhaled corticosteroids within the previous 90 days.

CONCLUSIONS: People with asthma receiving inhaled corticosteroids are at an increased risk of pneumonia or lower respiratory infection, with those receiving higher doses being at greater risk. Pneumonia should be considered as a possible side effect of inhaled corticosteroids, and the lowest possible dose of inhaled corticosteroids should be used in the management of asthma. (McKeever et al. ${ }^{7}, 2013$, p. 1788; Reprinted with permission of American College of Chest Physicians)

Use of ICS in chronic obstructive pulmonary disease patients has been demonstrated to be associated with increased risk of pneumonia. However, the risk of pneumonia related to the use of ICS in asthma is not known. To clarify this issue, a case-control study was carried out. As a result, the ICS usage has been found to be in dose-response relationship with the risk of pneumonia. In the group with the highest dose of ICS, the relative risk of pneumonia has been 2.04 compared to the patients with asthma without ICS use.

Statin exposure is associated with decreased asthmarelated emergency department visits and oral corticosteroid use. Tse et al. ${ }^{8}$ Am J Respir Crit Care Med 2013; 188:1076-82

RATIONALE: Statins, or HMG-CoA reductase inhibitors, may aid in the treatment of asthma through their pleiotropic antiinflammatory effects.

OBJECTIVES: To examine the effect of statin therapy on asthma-related exacerbations using a large population-based cohort.

METHODS: Statin users aged 31 years or greater with asthma were identified from the Population-Based Effectiveness in Asthma and Lung population, which includes data from five health plans. Statin exposure and asthma exacerbations were assessed over a 24-month observation period. Statin users with a statin medication possession ratio greater than or equal to $80 \%$ were matched to non-statin users by age, baseline asthma therapy, site of enrollment, season at baseline, and propensity score, which was calculated based on patient demographics and Deyo-Charlson conditions. Asthma exacerbations were defined as two or more oral corticosteroid dispensings, asthmarelated emergency department visits, or asthma-related hospitalizations. The association between statin exposure and each of the three outcome measures was assessed using conditional logistic regression.

MEASUREMENTS AND MAIN RESULTS: Of the 14,566 statin users, 8,349 statin users were matched to a nonuser. After adjusting for Deyo-Charlson conditions that remained unbalanced after matching, among statin users, statin exposure was associated with decreased odds of having asthma-related emergency department visits (odds ratio [OR], 0.64; 95\% confidence interval [CI], 0.53-0.77; $\mathrm{P}<0.0001)$ and two or more oral corticosteroid dispensings ( $\mathrm{OR}, 0.90 ; 95 \% \mathrm{CI}, 0.81-0.99 ; \mathrm{P}=0.04$ ). There were no differences in asthma-related hospitalizations (OR, 0.91; 95\% CI, 0.66-1.24; $\mathrm{P}=0.52$ ).

CONCLUSIONS: Among statin users with asthma, statin exposure was associated with decreased odds of asthma-related emergency department visits and oral corticosteroid dispensings. (Tse et al. ${ }^{8}, 2013$, p. 1076; Reprinted with permission of the American Thoracic Society. Copyright (02014 American Thoracic Society. Official Journal of the American Thoracic Society)

3-Hydroxy-3-methyl-glutaryl-CoA (HMG-CoA) reductase inhibitors, so-called statins, are known to have a wide range of anti-inflammatory effects in addition to effects on lipid metabolism. And the therapeutic effect has been noted in a variety of chronic diseases. However, the relevance of asthma control with statins has never been identified in a large study. Researchers have analyzed the relationship between the dose of the statin and asthma worsening observed through this study. Taking statins was associated with a reduction in the emergency room visits due to asthma and reduction in oral steroid regimen.

Randomized placebo-controlled trial to evaluate chronic dosing effects of propranolol in asthma. Short et al. ${ }^{9}$ Am J Respir Crit Care Med 2013;187:1308-14

RATIONALE: Unblinded studies have shown improvements in airway hyperresponsiveness with chronic nadolol in steroidnaive patients with asthma.

OBJECTIVES: To assess the effects of chronic nonselective $\beta$-blockade as add-on to inhaled corticosteroids (ICS) in patients with asthma.

METHODS: A double-blind randomized placebo-controlled crossover trial of propranolol in patients with mild-to-moderate asthma receiving ICS was performed. Participants underwent a 6- to 8-week dose titration of propranolol or placebo as tolerated to a maximum of $80 \mathrm{mg}$ per day. Tiotropium was given for the first 4 to 6 weeks of each treatment period.

MEASUREMENTS AND MAIN RESULTS: Primary outcome was methacholine challenge. Secondary outcomes included histamine challenge, pulmonary function, mini-asthma quality of life questionnaire (mini-AQLQ), and asthma control questionnaire (ACQ). Eighteen patients completed (mean [SEM]): age, 36 (4) yr; FEV1\%, 93 (2); ICS, 440 (66) $\mu \mathrm{g} / \mathrm{d}$. No significant difference was observed in methacholine or histamine challenge after exposure to propranolol versus placebo. For methacholine challenge, the doubling dilution difference was 0.04 
( $95 \%$ confidence interval [CI], -0.56 to 0.63 ), $\mathrm{P}=0.89$. Albuterol recovery at 20 minutes after histamine challenge was partially attenuated by propranolol versus placebo: FEV1\% mean difference, 5.28 (95\% CI, 2.54-8.01), $\mathrm{P}=0.001$. After chronic $\beta$-blockade there was a small worsening in FEV1 \% predicted of $2.4 \%$ (95\% CI, -0.1 to 4.8 ), $\mathrm{P}=0.055$. No difference was found for ACQ or mini-AQLQ.

CONCLUSIONS: This is the first placebo-controlled study to assess the effects of chronic nonselective $\beta$-blockade in asthma, showing no significant effect of propranolol compared with placebo on either methacholine or histamine airway hyperresponsiveness and no change in ACQ or AQLQ. (Short et al. ${ }^{9}, 2013$, p. 1308; Reprinted with permission of the American Thoracic Society. Copyright (2014 American Thoracic Society. Official Journal of the American Thoracic Society)

Because of the potential to cause bronchospasm, betablockers generally are avoided in patients with asthma. However, some studies reported that using beta-blockers in patients with asthma can reduce airway hyperresponsiveness. To investigate the effects of chronic administration of noncardioselective beta-blockers (propranolol) in asthmatics using ICS, the authors performed a double-blind, randomized, placebo-controlled clinical trial. Along with results methacholine challenge test, which was the primary outcome, the histamine challenge test, asthma quality of life questionnaire, ACQ showed no differences in both groups.

\section{Conflicts of Interest}

No potential conflict of interest relevant to this article was reported.

\section{References}

1. Wenzel S, Ford L, Pearlman D, Spector S, Sher L, Skobieranda F, et al. Dupilumab in persistent asthma with elevated eosinophil levels. N Engl J Med 2013;368:2455-66.

2. Busse WW, Holgate S, Kerwin E, Chon Y, Feng J, Lin J, et al. Randomized, double-blind, placebo-controlled study of brodalumab, a human anti-IL-17 receptor monoclonal antibody, in moderate to severe asthma. Am J Respir Crit Care Med 2013;188:1294-302.

3. Laviolette M, Gossage DL, Gauvreau G, Leigh R, Olivenstein R, Katial R, et al. Effects of benralizumab on airway eosinophils in asthmatic patients with sputum eosinophilia. J Allergy Clin Immunol 2013;132:1086-96.

4. Lin SY, Erekosima N, Kim JM, Ramanathan M, Suarez-Cuervo C, Chelladurai Y, et al. Sublingual immunotherapy for the treatment of allergic rhinoconjunctivitis and asthma: a systematic review. JAMA 2013;309:1278-88.

5. Sadatsafavi M, Lynd L, Marra C, Bedouch P, Fitzgerald M. Comparative outcomes of leukotriene receptor antagonists and long-acting beta-agonists as add-on therapy in asthmatic patients: a population-based study. J Allergy Clin Immunol 2013;132:63-9.

6. Wechsler ME, Laviolette M, Rubin AS, Fiterman J, Lapa e Silva JR, Shah PL, et al. Bronchial thermoplasty: long-term safety and effectiveness in patients with severe persistent asthma. J Allergy Clin Immunol 2013;132:1295-302.

7. McKeever T, Harrison TW, Hubbard R, Shaw D. Inhaled corticosteroids and the risk of pneumonia in people with asthma: a case-control study. Chest 2013;144:1788-94.

8. Tse SM, Li L, Butler MG, Fung V, Kharbanda EO, Larkin EK, et al. Statin exposure is associated with decreased asthmarelated emergency department visits and oral corticosteroid use. Am J Respir Crit Care Med 2013;188:1076-82.

9. Short PM, Williamson PA, Anderson WJ, Lipworth BJ. Randomized placebo-controlled trial to evaluate chronic dosing effects of propranolol in asthma. Am J Respir Crit Care Med 2013;187:1308-14. 